\title{
Características sociodemográficas y clínicas y antecedentes patológicos asociados a infección del tracto urinario en diabéticos
}

\author{
Sociodemographic, clinical and pathological characteristics associated \\ with urinary tract infection in diabetics: case and control study
}

Magno Victor Ríos-Quijano'

Ríos-Quijano MV. Características sociodemográficas y clínicas y antecedentes patológicos asociados a infección del tracto urinario en diabéticos. Rev Soc Peru Med Interna. 2019;32(I):9-14.

\begin{abstract}
RESUMEN
ОвлетIvo. Determinar qué características sociodemográficas y clínicas y antecedentes patológicos constituyen un mayor riesgo para tener infección del tracto urinario (ITU), según el estudio de casos/controles, en población diabética tipo 2. MATERIAL Y MÉtodos. Estudio comparativo, transversal, retrospectivo y explicativo realizado en el servicio de medicina interna del Hospital Loayza el año 2016. Se revisaron historias de 163 pacientes diabéticos con ITU y 163 diabéticos sin ITU. Se aplicó ji cuadrado inicialmente y luego regresión logística con estadístico de Wald para análisis multivariado. RESULTADOS. Las características sociodemográficas no tuvieron relación estadística con la ocurrencia de ITU $(p>0,05)$. EI tiempo de enfermedad $\geq 10$ años (OR=3,978 (IC95\% 1,467-10,790); $p=0,007)$, la insuficiencia renal crónica $(O R=8,412$ (IC95\% 1,832-38,615), $p=0,006)$, la litiasis renal (OR=10,604 (IC95\% 2,144-52,459), $p=0,004)$, la vejiga neurogénica $(O R=12,290$ (IC95\% 1,447-104,386), $p=0,022)$, el uso de corticoides (OR=17,850 (IC95\% 3,789-84,087), $p=0,000)$, la ITU previa $(O R=27,757(I C 95 \% 9,280-83,027), p=0,000)$ y la infección vaginal o la patología prostática (OR=4,958 (IC95\% 2,149-11,439), $p=0,000$ ) estuvieron asociados de forma independiente con la ocurrencia de ITU. ConcLusIón. Las características sociodemográficas no tienen relación estadística con ITU. Un tiempo de enfermedad de 10 años o más, la insuficiencia renal crónica, litiasis renal, vejiga neurogénica, uso de corticoides, ITU previa e infección vaginal o patología prostática están asociadas estadísticamente con la ocurrencia de ITU en la población de diabéticos tipo 2.

Palabras clave. Diabetes mellitus tipo 2, factor de riesgo, infección urinaria.
\end{abstract}

\begin{abstract}
OвJECTIVE. To determine which sociodemographic characteristics, clinical and pathological antecedents constitute a greater risk to have urinary tract infection (UTI), according to the case-control study, in type 2 diabetic population. Material and Methods. comparative, cross-sectional, retrospective study and explanatory, performed in the Internal medicine service of Loayza Hospital in 2016. Charts of 163 diabetic patients with UTI and 163 diabetics without UTI were reviewed. Chi square was initially applied and then logistic regression with Wald statistic for multivariate analysis. REsults. The sociodemographic characteristics have no statistical relationship with the occurrence of UTI $(p>0,05)$. Disease time $\geq 10$ years $(O R=3,978(95 \% C l 1,467-10,790), p=0,007)$, Chronic renal failure $(O R=8,412(95 \% \mathrm{Cl} \quad 1,832-38,615)$, $p=0,006)$, Kidney lithiasis $(O R=10,604(95 \% \mathrm{Cl} 2,144-52,459)$,
\end{abstract}

I Médico internista. Hospital Nacional Arzobispo Loayza. Universidad Nacional Mayor de San Marcos. $p=0,004)$, neurogenic bladder (OR $=12,290(95 \% \mathrm{Cl} 1,447$ $104,386), p=0,022)$, corticosteroid use $(O R=17,850(95 \% \mathrm{Cl}$ $3,789-84,087), p=0,000)$, previous UTI (OR=27,757 (95\% CI $9,280-83,027), \quad p=0,000)$ and present vaginal infection or prostatic pathology (OR $=4,958$ (95\% Cl 2,149-11,439), $p=0,000)$ were independently associated with the occurrence of ITUs. Conclusions. Sociodemographic characteristics have no statistical relationship with UTI. Disease time $>=10$ years, chronic renal failure, renal lithiasis, neurogenic bladder, use of corticosteroids, previous UTI and presenting vaginal infection or prostatic pathology are statistically associated with the occurrence of UTI in type 2 diabetic population.

KEYWORDS. Diabetes, risk factor, UTI.

\section{INTRODUCCIÓN}

La diabetes mellitus (DM) es un importante problema de salud pública y en las últimas décadas han aumentado sin pausa el número de casos y la prevalencia de la enfermedad. La prevalencia de la DM fue del 8,5\% 
entre los adultos mayores de 18 años, según la OMS, en su reporte del 2016, y una prevalencia de DM de 6,9\% en Perú. ${ }^{1}$

Los diabéticos son más propensos a las infecciones que sus pares no diabéticos. La infección del tracto urinario (ITU) es la más frecuente ${ }^{2,3}$ y puede llevar a insuficiencia renal ${ }^{4-6}$ o cuadros agudos graves como la pielonefritis enfisematosa.?

Surge la inquietud de saber porque no todos los diabéticos hacen infecciones urinarias. Debe haber situaciones o factores de riesgo asociados a un mayor riesgo de padecer ITU. Se mencionan el sexoo femenino, microalbuminuria, obesidad e insulinoterapia como factores de riesgo para desarrollar ITU, ${ }^{3}$ así como litiasis renal, insuficiencia renal crónica (IRC), vejiga neurogénica, padecer infección vaginal o patología prostática, antecedente de ITU previa ${ }^{8-14}$ y por el contrario algunos estudios reportan que la hemoglobina glicosilada (HbA1c), edad y duración de la DM no influyen en la incidencia de ITU, ${ }^{3}$ mientras que otros estudios por el contrario menciona que si están asociados a ocurrencia de ITU. ${ }^{8,10,12,15-19}$ El objetivo del estudio es determinar qué características sociodemográficas, clínicas y antecedentes patológicos en la población de diabéticos, constituyen un mayor riesgo para tener ITU, según estudio de casos y controles.

\section{MATERIAL Y MÉTODOS}

Estudio cuantitativo, comparativo, transversal, retrospectivo de casos y controles de una muestra de población de diabéticos mayores de 18 años, que acudieron al hospital Arzobispo Loayza, durante 2016.

Los criterios de inclusión fueron diabéticos tipo 2 atendidos en consulta externa o estuvieron hospitalizados en el servicio de medicina interna con o sin diagnóstico de ITU que conforman los grupos de casos y controles respectivamente. El diagnóstico de ITU fue clínico, por urocultivo y fue tratado. Los criterios de exclusión fueron diabéticos tipo 1, diabéticos con presencia de infección distinta a la ITU y con tratamiento antibiótico, historias clínicas incompletas, historias de diabéticos vistos en otros servicios y gestantes diabéticas.

La muestra fue obtenida de manera aleatoria simple, en dos grupos: casos (diabéticos con ITU) y controles (diabéticos sin ITU) con un nivel de confianza de $95 \%$ y un error del $5 \%$. Se obtuvo 326 pacientes, de los cuales 163 eran casos y 163 controles.
Se consignaron los datos demográficos, clínicos y antecedentes patológicos de las historias clínicas en una ficha de recolección de datos para casos y controles. Sobre la confiabilidad de la ficha, se obtuvo un coeficiente alfa de Crombach de 0,752 para los casos y 0,718 para los controles. Los resultados se presentan de manera descriptiva mediante frecuencia y porcentajes. Para el análisis bivariado, se buscó una aproximación mediante la prueba ji cuadrado, luego se aplicó el análisis multivariado de regresión logística, con recategorización de las variables, con el estadístico de Wald, para seleccionar qué variables contribuyen en la ocurrencia del evento. Un valor de $\mathrm{p}<0,05$ se aceptó como significancia. Se utilizó el programa estadístico SPSS v. 20.

\section{RESULTADOS}

De 326 diabéticos, los mayores de 40 años fueron los más afectados con $97,4 \%$. El $79,1 \%$ de pacientes

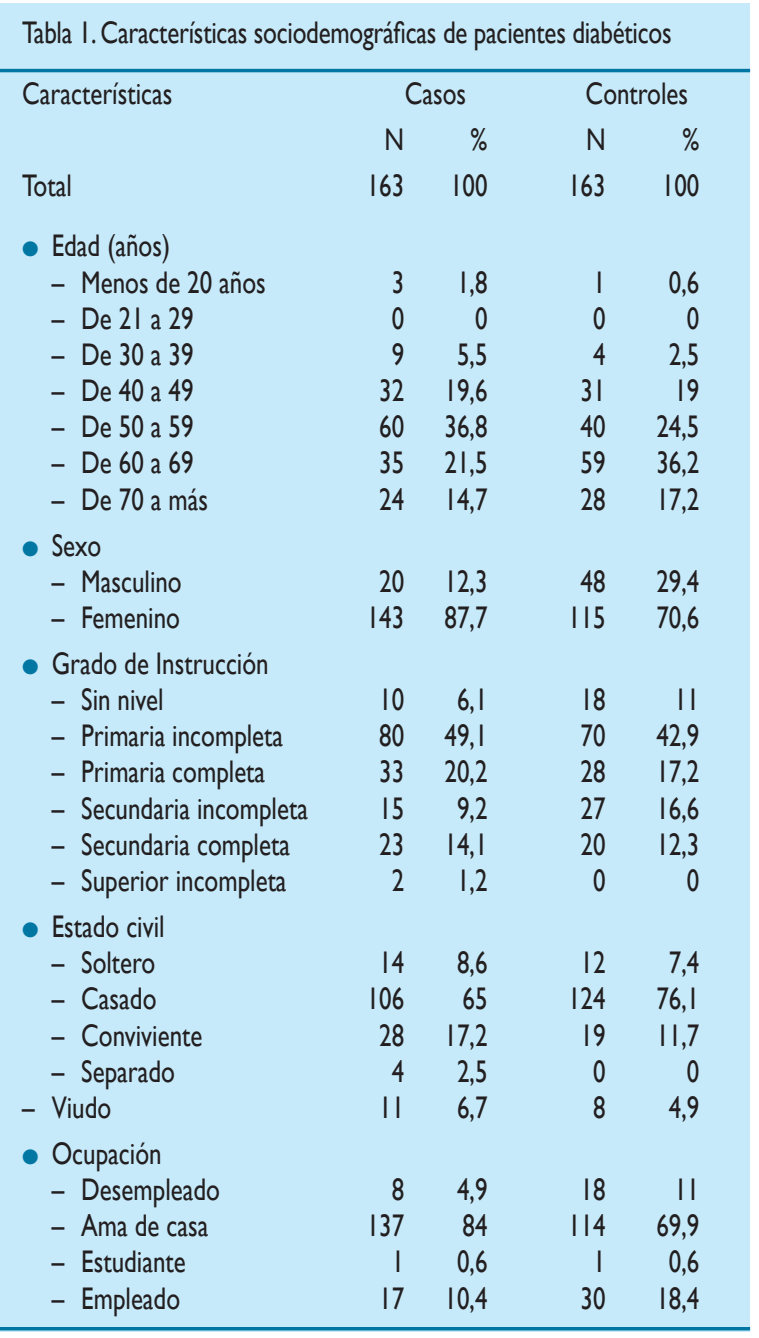




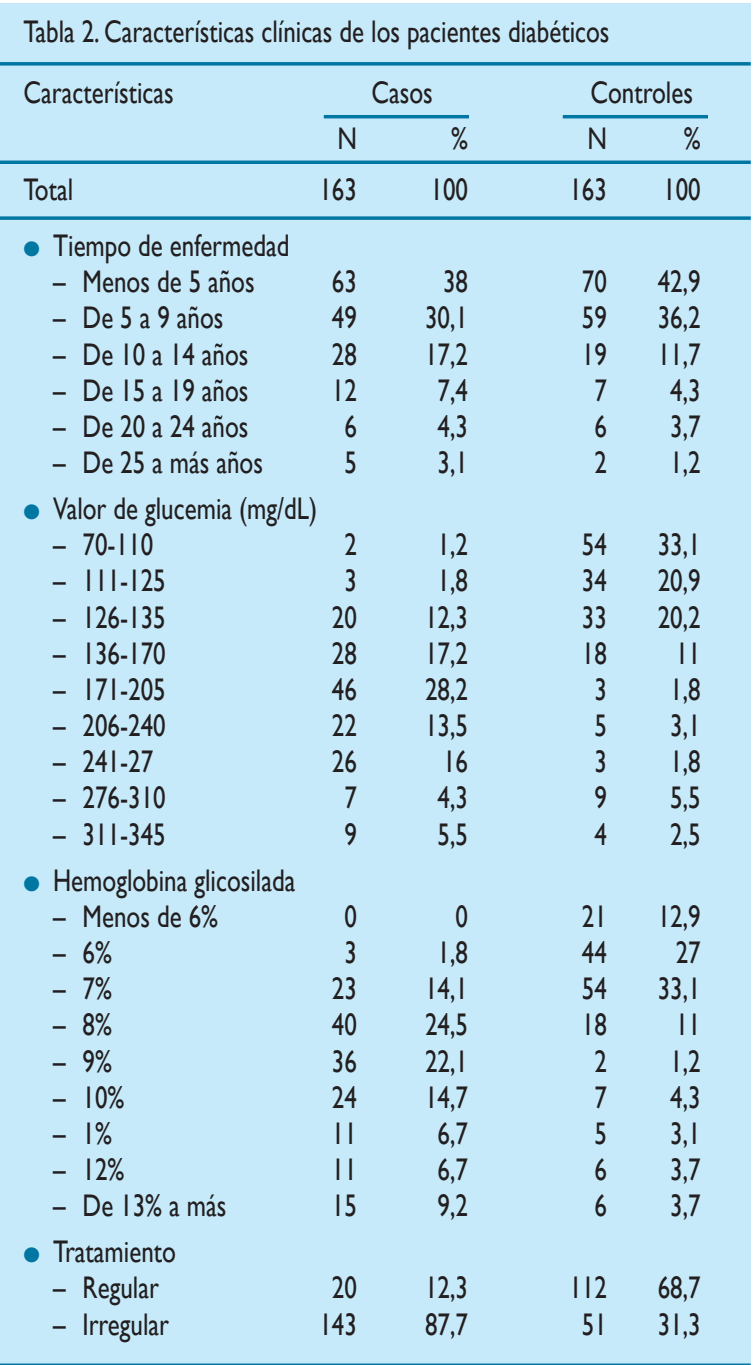

\begin{tabular}{|c|c|c|c|c|c|c|}
\hline \multirow[t]{2}{*}{ Antecedentes patológicos } & \multicolumn{2}{|c|}{ Total } & \multicolumn{2}{|c|}{ Sí } & \multicolumn{2}{|c|}{ No } \\
\hline & $\mathrm{N}$ & $\%$ & $\mathrm{~N}$ & $\%$ & $\mathrm{~N}$ & $\%$ \\
\hline \multicolumn{7}{|l|}{ - Casos } \\
\hline - Hipertensión arterial & 163 & 100 & 44 & 27 & 119 & 73 \\
\hline - Insuficiencia renal crónica & 163 & 100 & 25 & 15,3 & 138 & 84,7 \\
\hline - Litiasis renal & 163 & 100 & 33 & 20,2 & 130 & 79,8 \\
\hline - Vejiga neurogénica & 163 & 100 & 17 & 10,4 & 146 & 89,6 \\
\hline - Patología prostática' & 20 & 100 & 12 & 60 & 8 & 40 \\
\hline - Uso de corticoides & 163 & 100 & 28 & 17,2 & 135 & 82,8 \\
\hline - ITU previa & 163 & 100 & 154 & 94,5 & 9 & 5,5 \\
\hline - Infección vaginal previa ${ }^{2}$ & 143 & 100 & 84 & 58,7 & 59 & $4 I, 3$ \\
\hline - Cáncer & 163 & 100 & 5 & 3,1 & 158 & 96,9 \\
\hline \multicolumn{7}{|l|}{ - Controles } \\
\hline - Hipertensión arterial & 163 & 100 & 51 & 31,3 & 112 & 68,7 \\
\hline - Insuficiencia renal crónica & 163 & 100 & 5 & 3,1 & 158 & 96,9 \\
\hline - Litiasis renal & 163 & 100 & 4 & 2,5 & 159 & 97,5 \\
\hline - Vejiga neurogénica & 163 & 100 & 2 & 1,2 & 161 & 98,8 \\
\hline - Patología prostática' & 48 & 100 & 15 & 31,3 & 33 & 68,8 \\
\hline - Uso de corticoides & 163 & 100 & 4 & 2,5 & 159 & 97,5 \\
\hline - ITU previa & 163 & 100 & 41 & 25,2 & 122 & 74,8 \\
\hline - Infección vaginal previa ${ }^{2}$ & 115 & 100 & 12 & 10,4 & 103 & 89,6 \\
\hline - Cáncer & 163 & 100 & 0 & 0 & 163 & 100 \\
\hline
\end{tabular}

fueron mujeres y de este sexo era el 87,7\% de los casos y 70,6\% de los controles. A su vez, las amas de casa eran el 76,9\% del total de pacientes y el $84 \%$ de casos y $69,9 \%$ de controles.

Del total, 241 diabéticos $(73,9 \%)$ tuvieron un tiempo enfermedad menor de 10 años y de estos el 68,1\% fueron casos. El 98,2\% de casos tuvieron $\mathrm{HbA1c} \geq 7 \%$ y en los controles el 39,9\% tenía HbAlc $<7 \%$. El $87,7 \%$ de los casos tenían tratamiento irregular. En los casos, el $97 \%$ tenían glucosa $\geq 126 \mathrm{mg} / \mathrm{dL}$. En los casos, los antecedentes de ITU previa, patología prostática,

Tabla 4. Regresion Logistica para la ocurrencia de ITU según Caracteristicas Sociodemograficas

\begin{tabular}{|c|c|c|c|c|c|c|c|c|}
\hline \multirow[t]{2}{*}{ Variables explicativas } & \multirow[t]{2}{*}{ B } & \multirow[t]{2}{*}{ EE } & \multirow[t]{2}{*}{ Wald } & \multirow[t]{2}{*}{ gl } & \multirow[t]{2}{*}{ Sig (p) } & \multirow[t]{2}{*}{$\operatorname{Exp}(B)$} & \multicolumn{2}{|c|}{ IC95\% para EXP(B) } \\
\hline & & & & & & & $\overline{\text { Inferior }}$ & $\overline{\text { Superior }}$ \\
\hline $\begin{array}{l}\text { - Razón de las ventajas para edad } \\
\text { (menor de } 60 \text { años / de } 60 \text { años a más) }\end{array}$ & 0,489 & 0,45 & I,183 & 1 & 0,277 & $|, 63|$ & 0,676 & 3,935 \\
\hline $\begin{array}{l}\text { Razón de las ventajas para sexo } \\
\text { (mujer/hombre) }\end{array}$ & 2,461 & 2,164 & 1,294 & 1 & 0,255 & 11,717 & 0,169 & 813,905 \\
\hline $\begin{array}{l}\text { - Razón de las ventajas para grado de instrucción } \\
\text { (hasta primaria / de secundaria a más) }\end{array}$ & $-0,234$ & 0,529 & 0,196 & I & 0,658 & 0,791 & 0,281 & 2,23 \\
\hline $\begin{array}{l}\text { - Razón de las ventajas para estado civil } \\
\text { (otra condición / casado/conviviente) }\end{array}$ & 0,637 & 0,749 & 0,722 & I & 0,395 & I,89| & 0,435 & 8,214 \\
\hline $\begin{array}{l}\text { - Razón de las ventajas para ocupación } \\
\text { (ama de casa / otra ocupación) }\end{array}$ & 2,358 & 2,112 & 1,247 & I & 0,264 & 0,095 & 0,002 & 5,934 \\
\hline
\end{tabular}


Tabla 5. Regresión logística para la ocurrencia de infección del tracto urinario según características clínicas de la diabetes, antecedentes patológicos y variables explicativas

\begin{tabular}{|c|c|c|c|c|c|c|c|c|}
\hline \multirow[t]{2}{*}{ Variables explicativas } & \multirow[t]{2}{*}{ B } & \multirow[t]{2}{*}{ EE } & \multirow[t]{2}{*}{ Wald } & \multirow[t]{2}{*}{$g \mid$} & \multirow[t]{2}{*}{ Sig (p) } & \multirow[t]{2}{*}{$\operatorname{Exp}(B)$} & \multicolumn{2}{|c|}{ IC95\% para $\operatorname{Exp}(B)$} \\
\hline & & & & & & & $\overline{\text { Inferior }}$ & Superior \\
\hline \multicolumn{9}{|l|}{ - Según características clínicas de la diabetes } \\
\hline - Tiempo de enfermedad $\geq 10$ años & 1,103 & 0,5 & 4,873 & I & 0,027 & 3,015 & 1,132 & 8,03 \\
\hline - Valor de glucemia $\geq 125$ & $-1,98$ & 0,805 & 6,057 & I & 0,014 & 0,138 & 0,029 & 0,668 \\
\hline - Valor de hemoglobina glicosilada $\geq 7 \%$ & $-1,717$ & 1,028 & 2,791 & 1 & 0,095 & 0,18 & 0,024 & 1,346 \\
\hline - Tratamiento irregular & 0,455 & 0,509 & 0,799 & I & 0,371 & I,577 & 0,581 & 4,279 \\
\hline \multicolumn{9}{|l|}{ - Según antecedentes patológicos } \\
\hline - Hipertensión arterial & $-0,209$ & 0,414 & 0,255 & I & 0,614 & 0,812 & 0,361 & 1,826 \\
\hline - Insuficiencia renal crónica & 1,97 & 0,671 & 8,633 & I & 0,003 & 7,172 & 1,927 & 26,695 \\
\hline - Litiasis renal & 2,795 & 0,8 & 12,217 & I & 0 & 16,358 & $3,4 \mid 3$ & 78,402 \\
\hline - Vejiga neurogénica & 2,795 & 0,944 & 8,777 & I & 0,003 & $|6,37|$ & 2,576 & 104,053 \\
\hline - Uso de corticoides & 2,575 & 0,771 & 11,162 & I & 0,001 & 13,129 & 2,899 & 59,46 \\
\hline - Infección del tracto urinario previa & 3,742 & 0,519 & 51,985 & I & 0 & 42,177 & 15,252 & 116,635 \\
\hline - Presentó infección vaginal o patología prostática & 1,465 & 0,374 & 15,366 & I & 0 & 4,326 & 2,08 & 8,997 \\
\hline - Constante & $-3,933$ & 0,551 & 51,039 & I & 0 & 0,02 & & \\
\hline \multicolumn{9}{|l|}{ - Según variables explicativas } \\
\hline - Tiempo de enfermedad $\geq 10$ años $\left(X_{I}\right)$ & |,38| & 0,509 & 7,356 & I & 0,007 & 3,978 & $\mathrm{I}, 467$ & 10,79 \\
\hline - Valor de glicemia $\geq 125\left(X_{2}\right)$ & $-3,053$ & 0,698 & 19,117 & I & 0 & 0,047 & 0,012 & 0,186 \\
\hline - Insuficiencia renal crónica $\left(X_{3}\right)$ & 2,13 & 0,778 & 7,502 & I & 0,006 & 8,412 & 1,832 & 38,615 \\
\hline - Litiasis renal $\left(X_{4}\right)$ & 2,361 & 0,816 & 8,38 & I & 0,004 & 10,604 & 2,144 & 52,459 \\
\hline - Vejiga Neurogénica(X5) & 2,509 & 1,092 & 5,283 & I & 0,022 & 12,29 & I,447 & 104,386 \\
\hline - Uso de corticoides $\left(X_{6}\right)$ & 2,882 & 0,791 & 13,283 & I & 0 & 17,85 & 3,789 & 84,087 \\
\hline - Infección del tracto urinario previa $\left(X_{7}\right)$ & 3,323 & 0,559 & 35,345 & I & 0 & 27,757 & 9,28 & 83,027 \\
\hline - Presentó infección vaginal o patología prostática $\left(X_{8}\right)$ & $|, 60|$ & 0,427 & 14,084 & 1 & 0 & 4,958 & 2,149 & 11,439 \\
\hline - Constante & $-3,626$ & 0,614 & 34,844 & I & 0 & 0,027 & & \\
\hline
\end{tabular}

B: parámetro estimado EE: error estándar Exp(B): estimación de odds ratio (OR)

infección vaginal previa, litiasis renal, uso corticoides, IRC y vejiga neurogénica estuvieron presentes en el $94,5 \%, 60 \%, 58,7 \%, 20,2 \%, 17,2 \%, 15,3 \%$ y $10,4 \%$, respectivamente. En el grupo de controles, los antecedentes de HTA, patología prostática, ITU previa e infección vaginal previa estuvieron presentes en el $31,3 \%, 31,3 \%$ y $10,4 \%$, respectivamente. Dentro de las característica sociodemográficas y con variables recategorizadas se aplicó el análisis multivariado de regresión logística, obteniéndose un valor de $\mathrm{p}>0,05$ con el estadístico de Wald en todas ellas, es decir, no tienen asociación estadística con la ocurrencia de ITU.

Dentro de las características clínicas de la DM y con variables recategorizadas se aplicó el análisis multivariado de regresión logística. Se obtuvo un valor de $\mathrm{p}<0,05$ solo para la variable tiempo de enfermedad mayor o igual a 10 años, estando entonces asociado estadísticamente a la ocurrencia de ITU.

Dentro de los antecedentes patológicos, se aplicó el análisis multivariado de regresión logística. Se obtuvo un valor de $\mathrm{p}<0,05$ para la IRC, litiasis renal, vejiga neurogénica, uso de corticoides, ITU previa y presentar patología prostática o infección vaginal, es decir, tienen asociación estadística con la ocurrencia de ITU. Tabla 5. Finalmente, se realizó el análisis multivariado de regresión logística tomando en cuenta solo las variables que habían tenido significancia estadística (variables explicativas) tanto de las características clínicas de la DM como los antecedentes patológicos, en un modelo final, obteniéndose un valor de $\mathrm{p}<0,05$ para el tiempo de enfermedad mayor o igual a 10 años, IRC, litiasis renal, vejiga neurogénica, uso de corticoides, ITU previa y presentar patología prostática o infección vaginal. El valor de glicemia $\geq 125$ es un factor de protección para la ocurrencia de ITU, puede verse en la Tabla 5. Así mismo, se calculó el valor de $\mathrm{R}$ cuadrado de Nagelkerke, que es una versión corregida de la $\mathrm{R}$ cuadrado de Cox y Snell, de 0,767. Esto significa que el $76,7 \%$ de la variación de la variable dependiente (ITU en diabéticos) es explicada por las variables explicativas consideradas en Resumen del modelo. 


\section{DISCUSIÓN}

En el presente estudio, las características sociodemográficas no tuvieron asociación con la ocurrencia de ITU luego de aplicar regresión logística. Respecto a las características clínicas de la DM, el análisis por regresión logística evidenció solo la enfermedad mayor igual a 10 años de evolución con la ocurrencia de ITU. Se encontró $73,9 \%$ de diabéticos con un tiempo de enfermedad menor a 10 años, lo que contrasta con los datos de estudios como el de Geerlings y col., ${ }^{4}$ el año 2000, en Países Bajos, donde en un grupo de 589 pacientes diabéticas, han reportado un tiempo de enfermedad promedio de 13,7 $\pm 11,3$ años. Tapia y col., ${ }^{8}$ en 2001, en un estudio en el Hospital Cayetano Heredia en Lima, han reportado un tiempo de enfermedad de 11,398 \pm 9,14 años de evolución en 210 pacientes diabéticos. En Managua, en 2016, Bordas $^{21}$ ha comunicado en 230 pacientes diabéticos, que 46,1\% tenían más de 10 años de enfermedad. Chirinos, ${ }^{16}$ en 2016, en Trujillo, ha reportado que, en 300 pacientes diabéticos estudiados, $60 \%$ tenían más de 10 años de enfermedad. A su vez, Alebiosu y col., ${ }^{21}$ en Nigeria, en 2003, han descrito la duración de la DM $(\mathrm{p}<0,001)$ como factor de riesgo para ITU sintomática y septicemia. Boyko y col., ${ }^{17} \mathrm{en}$ 2002, han descrito una duración mayor de 10 años como factor de riesgo para ITU sintomática entre mujeres diabéticas posmenopaúsicas y, en 2005, en un estudio en EE.UU., han comunicado la asociación de ITU con diabéticos de más de 10 años de evolución $(\mathrm{RR}=2,6$, IC95\%: 1,3, 5,1). ${ }^{22}$ Janifer y col., ${ }^{12}$ en 2009, Geerlings y col..${ }^{14}$ en 2013, Chirinos ${ }^{16}$ y Bordas, ${ }^{20}$ ambos en 2016, han reportado los mismos hallazgos. Solo Guang-Dan y col. ${ }^{18}$ han descrito en un metaanálisis, realizado en 2015, en China, que la duración mayor de 5 años de DM2 tiene un riesgo significativo para desarrollar infecciones en diabéticos. Esta discordancia con nuestros hallazgos podrían deberse a un diagnóstico más temprano, un acceso mejor a los servicios de salud y por ser el Hospital Loayza, un hospital que atiende pacientes de todo el país.

Por otro lado, en el presente estudio ni el valor de la glicemia ni valor de HbA1c estuvieron asociados con el desarrollo de ITU, tal como lo han descrito Geerlings y col., ${ }^{4}$ el año 2000 en Países Bajos; de igual manera, Bokyo y col., ${ }^{22}$ en EE.UU., en 2005, y Raoofi y col., ${ }^{19}$ en Irán, en 2013. Así mismo, Geerlings y col. ${ }^{14}$ han reportado, en 2013, no haber encontrado asociación entre los niveles de HbA1c o glicemia con el desarrollo de ITU en diabéticos tipo 2, tal como lo han descrito Nitzan y col., ${ }^{19}$ en un estudio, en 2015, en Reino Unido. Sin embargo, Aswani y col., ${ }^{23}$ en 2014 , han reportado un valor de HbA1c elevado como factor de riesgo para ITU $(p<0,001)$ entre 181 diabéticos, mientras que Gaung-Dan y col., ${ }^{18}$ en 2015, en China, han descrito que el control adecuado de la glucosa se asocia a un menor riesgo de ITU estadísticamente significativo. Finalmente, Janifer y col., ${ }^{12}$ en la India, en el año 2009, han destacado que un pobre control de la glicemia está asociado significativamente con la ITU en diabéticos. Estas discrepancias podrían deberse a diferencias de las características de los diabéticos incluidos en cada estudio, por lo que es necesario realizar otros estudios adicionales.

Respecto a los antecedentes patológicos de los pacientes, los hallazgos coinciden con el de la literatura; $\mathrm{Hu}$ y col.. ${ }^{10}$ en 2004, han concluido que la historia de ITU previa y DM en tratamiento en mujeres posmenopáusicas se asoció con un mayor riesgo de ITU, Janifer y col., ${ }^{12}$ en 2009, han descrito que la historia previa de ITU está asociada significativamente a ITU en diabéticos; Geerlings y col., ${ }^{14}$ en 2014, han mencionado que la ITU previa seis meses antes es un factor de riesgo de ITU en DM2. Varios estudios han evaluado la prevalencia de infecciones ginecológicas en la DM, en especial candidiasis vaginal, la cual predispone a ITU, ${ }^{14,15}$ tal como lo han mencionado Hu y col. ${ }^{10}$

La nefropatía diabética establecida, descrita por $\mathrm{Hu}$, y la insuficiencia renal crónica, mencionada por Martin ${ }^{9}$ son factores de riesgo para ITU en diabéticos. Tapia y col., ${ }^{8}$ en 2001, han destacado la asociación estadísticamente significativa entre vejiga neurogénica y presencia de ITU en diabéticos en un hospital de Lima. Brown y col., ${ }^{11}$ en 2005, han descrito que el $50 \%$ de mujeres con DM tienen disfunción vesical, como vejiga neurogénica, lo cual es un importante factor de riesgo para ITU. A su vez, Armas y col., ${ }^{24}$ en un estudio Trujillo, en 2007, han concluido que la vejiga neurogénica constituye un factor predisponente de mayor relevancia para ITU sintomática en pacientes con DM2. Por otro lado, Peleg y col., ${ }^{25}$ en el mismo año 2007, han reportado que la vejiga neurogénica es un factor de riesgo para ITU en diabéticos.

Martin ${ }^{9}$ ha mencionado que la inmunosupresión ya sea por enfermedad autoinmune frecuentemente asociada a DM, corticoterapia o tratamiento inmunosupresor es 
un factor probado de riesgo para infecciones y dentro de ellas, la ITU; asimismo, indica que la obstrucción al flujo urinario como la patología prostática (hipertrofia prostática benigna y cáncer de próstata) en diabéticos predispone a ITU. Así mismo, Nitzan y col., ${ }^{13}$ en 2015, en Reino Unido, han descrito que en varones diabéticos la prostatitis aguda o crónica y la biopsia de próstata incrementa riesgo de ITU. Martin ${ }^{9}$ ha destacado que la litiasis renal en pacientes diabéticos, como a cualquier otro paciente que lo padezca, es un factor de riesgo para ITU.

Las limitaciones del estudio serían la predominancia de la población femenina en las atenciones de todas las especialidades del hospital, y el no haber considerado diabéticos atendidos en otras especialidades.

En conclusión, no se encontró relación entre las características sociodemográficas con la ocurrencia de ITU. Diabéticos con mayor o igual a 10 años de evolución con antecedente de insuficiencia renal crónica, litiasis renal, vejiga neurogénica, uso de corticoides, ITU previa y presentar infección vaginal o patología prostática están en mayor riesgo estadístico de padecer ITU.

\section{REFERENCIAS BIBLIOGRÁFICAS}

I. Organización Mundial de la Salud. Informe mundial sobre la diabetes. 2016

2. Patterson JE,AndrioleVT. Bacterial urinary tract infections in diabetes. Infect Dis Clin North Am. 1997; I (3):735-50.

3. Al-Rubeaan KA, Moharram O, Al-Naqeb D, Hassan A, Rafiullah MR. Prevalence of urinary tract infection and risk factors among saudi patients with diabetes. World J Urology. 2013;31:573-578.

4. Geerlings SE, Stolk RP, Camps MJ, et al. Diabetes Women Asymptomatic Bacteriuria Utrecht Study Group: Risk factors for symptomatic urinary tract infection in women with diabetes. Diabetes Care. 2000; 23:1737-174I.

5. Chia T,Timar B, Muntean D, Baditoiu L, Horhat F, Hogea E, et al. Urinary tract infections in romanian patients with diabetes: prevalence, etiology, and risk factors. Therap Clin Risk Manag. 2017;13: I-7.

6. Pallares J, López A, Cano A, Fábrega J, Mendive J. La infección urinaria en el diabético.Aten Primaria 1998;21:630-7.

7. Ríos M,Pamo O,Alvarado A, Cumpa R, Cruzado C. Pielonefritis enfisematosa. Rev Soc Peru Med Interna. 20I2;25(3).
8. Tapia GG, Chirinos JL, Tapia LM, Factores contribuyentes al desarrollo de infecciones adquiridas en la comunidad en diabéticos tipo 2 admitidos en salas de medicina del Hospital Nacional Cayetano Heredia. Cad. Saúde Pública, Rio de Janeiro, nov-dez, 2001;17(6):1357-1365.

9. Martin JC. Infecciones urinarias complicadas: revisión y tratamiento. Información Terapéutica del Sistema Nacional de Salud. 2004;28(6):I37-I44.

10. Hu KK, Boyko EJ, Scholes D, Normand E, et al. Risk factors for urinary tract infections in postmenopausal women.Arch Intern Med. 2004; 164:989-9.

II. Brown J,Wessells H, Chancellor M, Howards S, Stamm W, Stapleton A, et al. Urologic complications of diabetes. Diabetes Care. 2005;28(I):I77-I85.

12. Janifer J, Geethalakshmi S, et al. Prevalence of lower urinary tract infection in south indian type 2 diabetic subjects. Indian J Nephrol. 2009; I9(3): I07-I I.

13. Nitzan O, Elias M, Chazan B, SalibaW. Urinary tract infections in patients with type 2 diabetes mellitus: review of prevalence, diagnosis, and management. Diabetes Metab Syndr Obes. 2015;8:129-136.

14. Geerlings S, Fonseca V, Castro-Diaz D, List J, Parikh S. Genital and urinary tract infections in diabetes: Impact of pharmacologically-induced glucosuria. Diabetes Res Clin Pract. 2014;103(3):373-38I.

15. Hirji I,Andersson S, Guo Z, Hammar N, Gomez-Caminero A. Incidence of genital infection among patients with type 2 diabetes in the UK General Practice Research Database.J Diabetes Complicat. 2012;26:501-505.

16. Chirinos $\mathrm{QH}$. Factores asociados a infección de vías urinarias en adultos con diabetes mellitus tipo 2. [tesis]. Trujillo. Universidad Privada Antenor Orrego. Facultad de Medicina Humana. 2016.

17. Boyko EJ, Fihn SD, Scholes D, Chen CL, Normand EH, Yarbro P. Diabetes and the risk of acute urinary tract infection among postmenopausal women. Diabetes Care. 2002;25:1778-83.

18. Guang-Dan Z, Jia-Ying S, Ming-Jing Z, Ling-Ling W, Fang-Zhi L, Shuo L, et al. Risk factors of community-associated infections in chinese patients with diabetes: a meta-analysis. J Transl Int Med. 2015; 3(I): 17-23.

19. Raoofi A, Ghavami M, Shahhamzeh M, et al. The impact of demographic factors and blood sugar control on the incidence of urinary tract infections in Khorramabad in 2013. Iran Red Crescent Med J. 2016; I8(5)e21942.

20. Bordas NB. Causas de hospitalización de pacientes diabéticos tipo 2 en medicina interna del hospital alemán nicaragüense de enero a diciembre de 2015. [Tesis]. Nicaragua: Universidad Nacional Autónoma de Nicaragua. 2016.

21. Alebiosu CO,Osinupebi OA, Olajubu FA.Significant asymptomatic bacteriuria among nigerian type 2 diabetics. J Nat Med Assoc. 2003;95(5):344-349.

22. Boyko EJ, Fihn SD, Scholes D, Abraham L, Monsey B. Risk of urinary tract infection and asymptomatic bacteriuria among diabetic and non-diabetic postmenopausal women. Am J Epidemiol. 2005; 161:557-64.

23. Aswani SM, Chandrashekar U, Shivashankara K, et al. Clinical profile of urinary tract infections in diabetics and non-diabetics.Australasian Med J. 2014;7:29-34.

24. Armas L, Gavidia A. Etiología y susceptibilidad antimicrobiana de bacteriuria sintomática en pacientes mujeres con diabetes mellitus tipo 2. Rev Méd Trujillo 2013;9(I).

25. Peleg AY, Weerarathna T, McCarthy JS, Davis TM. Common infections in diabetes: pathogenesis, management and relationship to glycaemic control. Diabetes Metab Res Rev. 2007;23(I):3-13.

CORRESPONDENCIA: Magno Víctor Ríos Quijano mvrq@yahoo.es

DECLARACIÓN DE CONFLICTO DE INTERÉs: Ninguno, según el autor.

FINANCIAMIENTO: Fue asumido en su totalidad por el autor.

FECHA DE RECEPCIÓN: 29 de enero de 2019.

FECHA DE ACEPTACIÓN: I 5 de marzo de 2019. 